\title{
A Case of Phaeohyphomycosis Caused by Exophiala oligosperma Successfully Treated with Local Hyperthermia
}

\author{
Tatsuo Fukai ${ }^{1}$, Masataro Hiruma ${ }^{2}$, Yumi Ogawa ${ }^{1}$, Shigaku Ikeda ${ }^{1}$, Hiroshi Ikeda ${ }^{3}$, \\ Ayako Sano ${ }^{4}$, Koichi Makimura ${ }^{5}$ \\ ${ }^{1}$ Department of Dermatology and Allergology, Juntendo University Graduate School of Medicine \\ ${ }^{2}$ Department of Dermatology and Allergology, Juntendo University Nerima Hospital \\ ${ }^{3}$ Department of Dermatology, Kameda Medical Center \\ ${ }^{4}$ Department of Animal Sciences, Faculty of Agriculture, University of the Ryukyus \\ ${ }^{5}$ Institute of Medical Mycology, Teikyo University
}

\begin{abstract}
A 58-year-old Japanese woman who was engaged in dairy farming presented with multiple subcutaneous nodules and abscesses on the dorsum of her left hand from 5 months ago. These had been unsuccessfully treated with oral itraconazole. The patient had a history of Sjögren syndrome and diabetes mellitus, for which she had been taking oral prednisolone for 10 years. Direct microscopy of a pus sample treated with potassium hydroxide $(\mathrm{KOH})$ showed brownish-red branching hyphae. In fungal culture, black colonies covered with gray-white villi were formed. Slide culture showed conidiogenesis from an annellide. The fungal strain was identified as Exophialia oligosperma by molecular biological techniques. Histopathological examination revealed abscesses and surrounding granulomatous infiltration in the dermis and subcutis, and hyphae in the granulomatous infiltration in the outer area. However, no eumycotic granules were observed. The diagnosis was phaeohyphomycosis caused by $E$. oligosperma. Since the previous treatment with itraconazole had not been effective, we performed daily hyperthermia using a disposable body warmer and drainage of the pus, which ceased after 3 weeks. After approximately 4 months, the skin eruptions became scarred. To the best of our knowledge, this is the second case of phaeohyphomycosis caused by E. oligosperma reported in Japan which was successfully treated with hyperthermia.
\end{abstract}

Key words : phaeohyphomycosis, Exophiala oligosperma, local hyperthermia

\section{Introduction}

Dematiaceous fungal infections are classified into chromoblastomycosis, phaeohyphomycosis, eumycetoma and others according to morphological characteristics of the fungal elements and clinical features. Phaeohyphomycosis is caused by fungi such as Fonsecaea pedrosoi, Exophiala dermatitidis, Exophiala jeanselmei and others that cause opportunistic infection secondary to trauma in immunocompromised patients and produce subcutaneous nodules and abscesses.

Here, we report our recent experience of a case of phaeohyphomycosis in a patient with a history of connective tissue disease who was successfully treated with local hyperthermia. To the best of our knowledge, this is the second case of phaeohyphomycosis caused by E. oligosperma reported in Japan. 


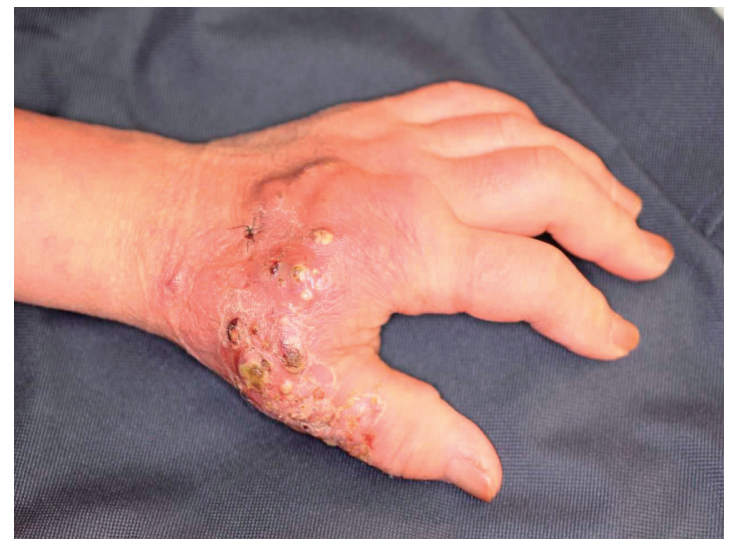

Fig. 1. Clinical findings at the first examination: multiple subcutaneous nodules and abscesses are observed on the thumb and central dorsum of the left hand, which were partly ruptured and discharged pus.

\section{Case report}

In October, 2011, a 58-year-old Japanese woman who was engaged in dairy farming presented with multiple nodules on the dorsum of her left hand. At initial presentation 5 months earlier, a dermatologist had diagnosed the nodules as phaeohyphomycosis based on fungal culture and histopathological findings. The patient had received treatment with itraconazole (ITCZ) $200 \mathrm{mg} /$ day for 1 month; however, the eruptions persisted. The patient had a history of Sjögren syndrome, diabetes mellitus, chronic renal failure, and atrial fibrillation and had received treatment with prednisolone for approximately 10 years.

At the first examination in our hospital, multiple subcutaneous nodules and abscesses were observed on the thumb and central dorsum of her left hand which were partly ruptured and discharged pus. No obvious disturbance of motility was observed (Fig. 1). No similar significant eruption was present in the left upper extremity or in any other parts of the body. The left axillary lymph nodule was not palpable. CT scan of the neck, chest, abdomen, and pelvis and magnetic resonance imaging of the head revealed no evidence of lesions in other organs.

There were no abnormal findings in complete blood count and blood biochemical tests, although an increased serum 1, 3- $\beta$-D-glucan level (436pg/ $\mathrm{m} /$ ) was noted.

Mycological analysis: A pus sample was taken from abscesses of the dorsum of her left hand and treated with potassium hydroxide $(\mathrm{KOH})$ for

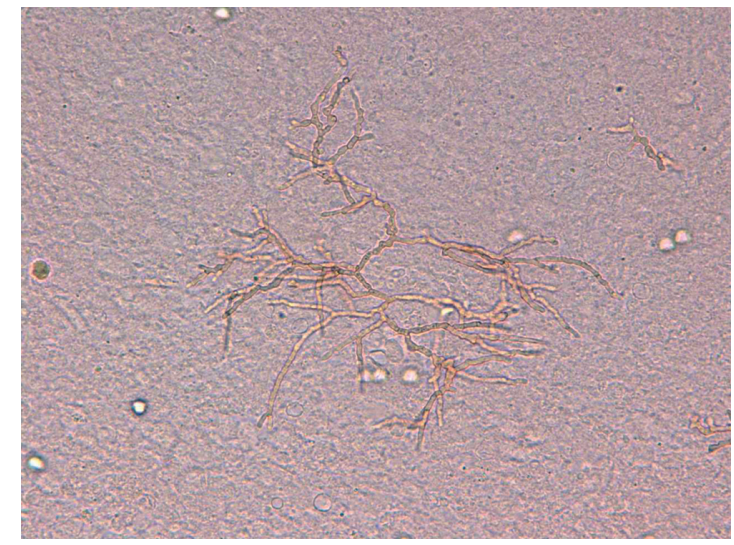

Fig. 2. A potassium hydroxide-treated pus sample: brownish-red branching hyphae are observed.

microscopic analysis. Direct microscopy of the sample revealed brownish-red branching hyphae (Fig. 2).

Pus and skin specimens taken from nodules of the dorsum of her left hand were cultured on a Mycosel agar slant medium, producing black colonies in 1-2 weeks. The colonies were then cultured to produce giant cells using a potato dextrose agar (PDA) medium and a Sabouraud's dextrose agar (SDA) medium. After 4 weeks, colonies covered with short villi of gray-white hyphae had formed, with diffused brown pigment in the surrounding area (Figs. 3 and 4). Slide culture showed conidiogenesis from an annellide (Fig. 5). We concluded that the causative agent might belong to Exophiala spp.

We later measured the minimum inhibitory concentration (MIC) of this strain. As shown in Table 1, the strain was somewhat sensitive to amphotericin B (MIC: $0.5-1$ ), but not to other major antifungal agents.

Molecular analysis: To identify the strain, we isolated DNA from the colonies and performed sequence analysis of the internal transcribed spacer (ITS) region of ribosomal DNA, using methods previously described ${ }^{1,2)}$. It was identified as $E$. oligosperma, and we registered it with accession number AB777520 in the DNA Data Bank of Japan.

Histopathological analysis: Histopathological examinations of skin specimens from earlier biopsies of nodules of her left hand revealed abscesses and surrounding granulomatous infiltration in the dermis and subcutis, and fibrosis in the outer area. Many fungal elements, consisting partly of hyphae, were observed in the area 


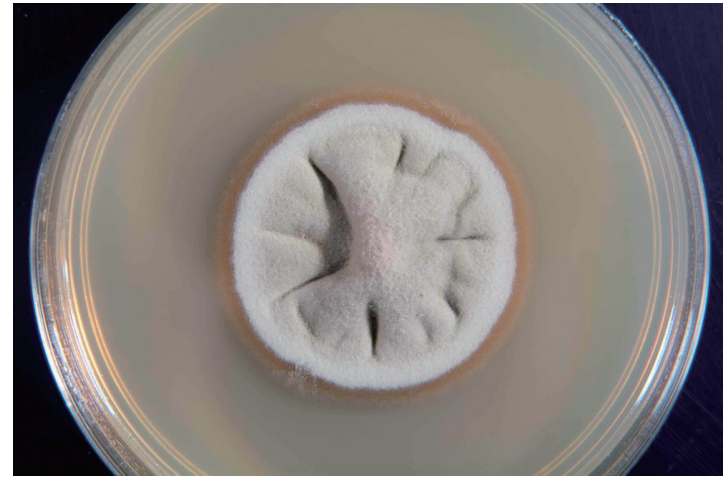

Fig. 3. Giant cells cultured on a potato dextrose agar medium at $25^{\circ} \mathrm{C}$ for 4 weeks: colonies covered with short villi of gray-white hyphae are formed and diffused brown pigment is observed in the surrounding area.

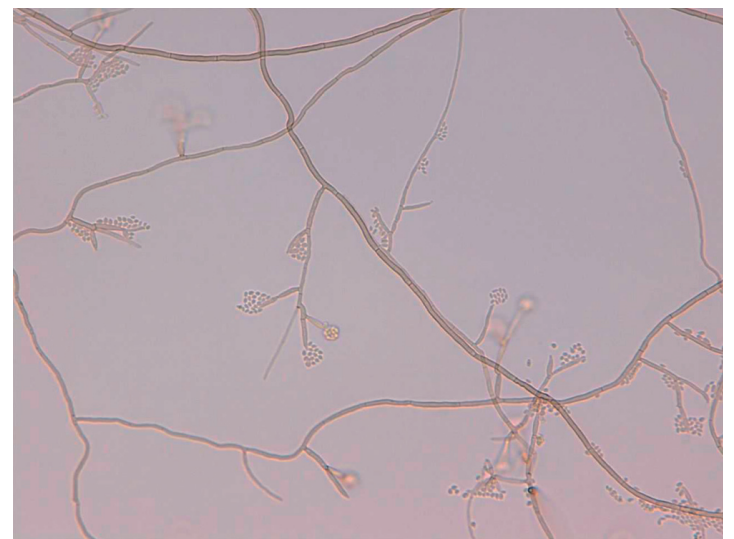

Fig. 5. Slide culture on a potato dextrose agar medium at $25^{\circ} \mathrm{C}$ for 4 weeks: Conidiogenesis from an annellide is observed.

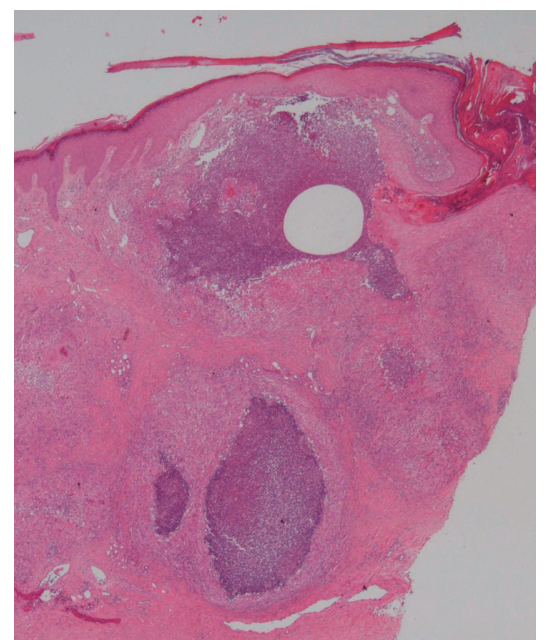

Fig. 6. Histopathological findings on hematoxylineosin stain (original magnification $\times 20$ ): Abscesses and surrounding granulomatous infiltration are observed in the dermis and subcutis.

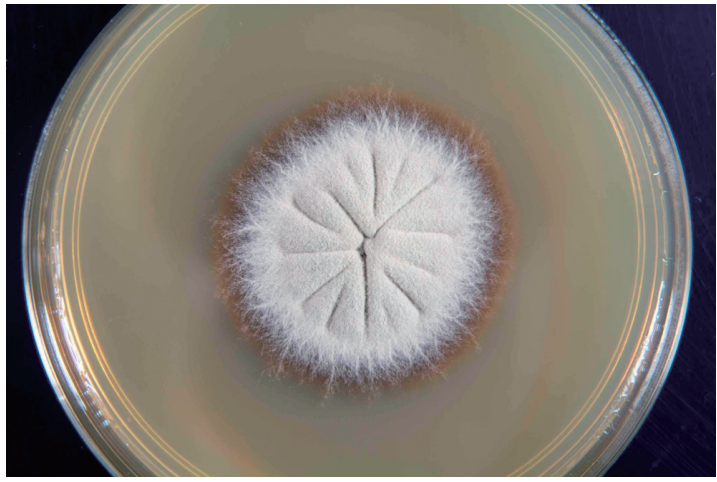

Fig. 4. Giant cells cultured on a Sabouraud's dextrose agar medium at $25^{\circ} \mathrm{C}$ for 4 weeks: colonies covered with short villi of gray-white hyphae are formed and diffused brown pigment is observed in the surrounding area.

Table 1. MICs of major antifungal agents

\begin{tabular}{ccccc}
\hline & \multicolumn{4}{c}{ MIC } \\
\cline { 2 - 5 } & $24 \mathrm{~h}$ & $48 \mathrm{~h}$ & $72 \mathrm{~h}$ & 7 day \\
\hline MCFG & $16 \uparrow$ & $16 \uparrow$ & $16 \uparrow$ & $16 \uparrow$ \\
AMPH-B & 0.5 & 1 & 1 & 1 \\
5 -FC & $64 \uparrow$ & $64 \uparrow$ & $64 \uparrow$ & $64 \uparrow$ \\
FCZ & $64 \uparrow$ & $64 \uparrow$ & $64 \uparrow$ & $64 \uparrow$ \\
ITCZ & 8 & $8 \uparrow$ & $8 \uparrow$ & $8 \uparrow$ \\
VRCZ & $8 \uparrow$ & $8 \uparrow$ & $8 \uparrow$ & $8 \uparrow$ \\
MCZ & 8 & 8 & 8 & 8 \\
\hline
\end{tabular}

around the abscesses and surrounding granulomatous infiltration, but there was no evidence of eumycotic granules (Figs. 6, 7 and 8). Consequently, the diagnosis was phaeohyphomycosis caused by $E$. oligosperma.

Since the previous treatment with itraconazole had not been effective, we chose a different treatment strategy. We drained the pus from the lesion and instructed the patient to apply disposable body warmers to the lesion every day for at least four hours, holding the body warmer in place by bandages. The nodules and abscesses rapidly decreased in size, and the discharge of pus ceased in approximately 3 weeks, leaving only comedos and subcutaneous abscesses (Fig. 9). Approximately 4 months after initiation of hyperthermia, only scars remained (Fig. 10). 


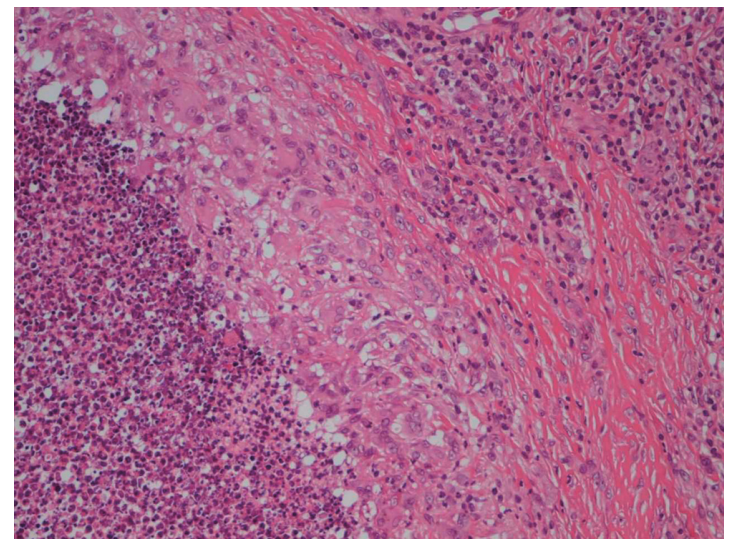

Fig. 7. Histopathological findings on hematoxylineosin stain (original magnification $\times 20$ ): Abscesses contain many neutrophils. Histiocytes, giant cells and lymphocytes are observed in the surrounding granulomatous infiltration. No evidence of eumycotic granules is observed.

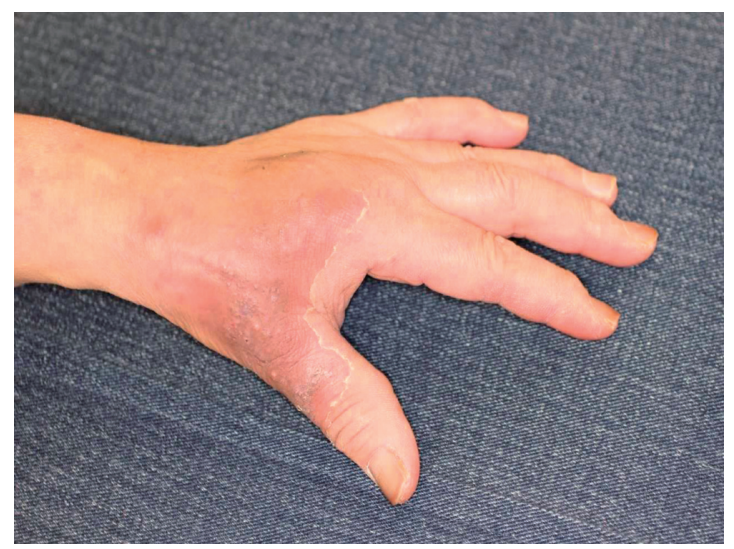

Fig. 9. Clinical findings after 3 weeks of local hyperthermia. After discharge of the pus, comedos and subcutaneous nodules remain.

\section{Discussion}

Phaeohyphomycosis is one of the dematiaceous fungal infections identified by Ajello ${ }^{3)}$. It is an opportunistic infection disease developed in compromised patients often through wound contamination with soil. The patient of the present report was engaged in dairy farming, which may have contributed to the cause of the infection.

The primary causative agent of phaeohyphomycosis is Exophiala spp. Advances in molecular biology have led to some changes in the classification of bacterial and fungal species, including Exophiala spp. Fungi that were previously classified as $E$. jeanselmei are now further

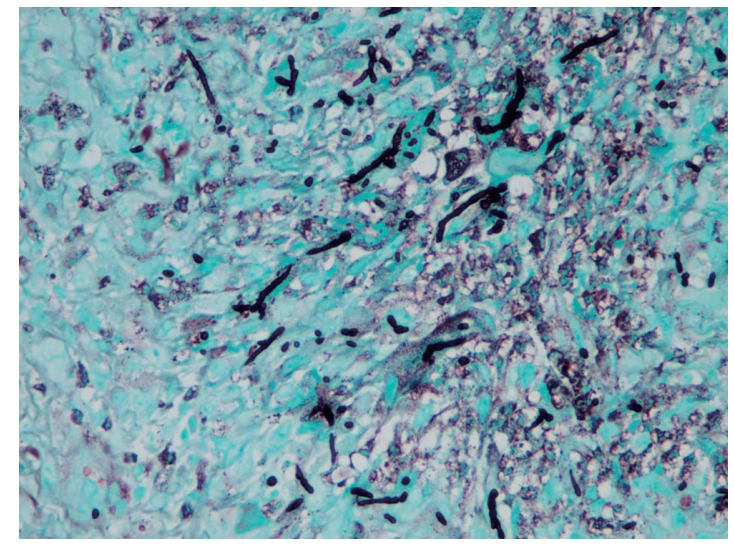

Fig. 8. Histopathological findings on Grocott stain (original magnification $\times 400$ ): Many fungal elements are observed mainly in the granulomatous infiltration surrounding the abscess.

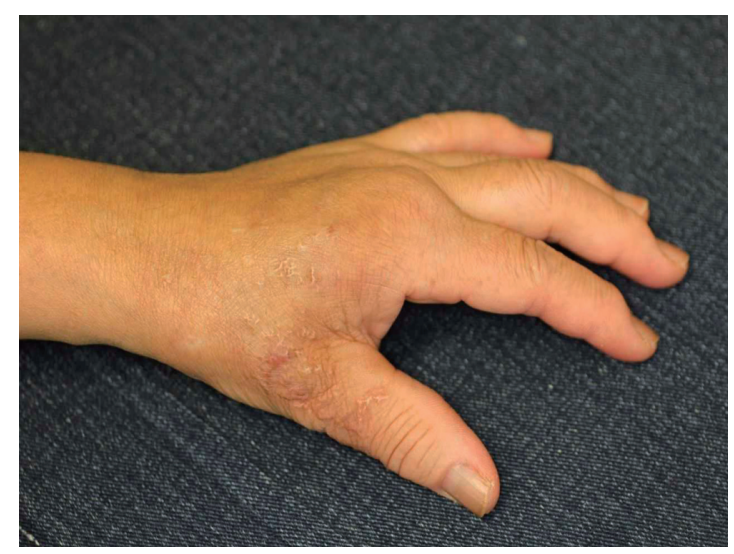

Fig. 10. Clinical findings after 4 months of local hyperthermia. Eruptions have disappeared, and only scars remain.

classified into several species, based on morphological features and other characteristics. In the case presented, sequence analysis of the ITS region identified the causative agent as E. oligosperma.

E. oligosperma is a fungal strain that was newly identified by de Hoog et al. in 2003. ${ }^{4)}$ To date, only 3 cases of deep dermatomycosis caused by this strain have been reported. ${ }^{5-7)}$ To the best of our knowledge, our is the second case reported in Japan (Table 2). Some cases of sepsis ${ }^{8)}$ and sinusitis ${ }^{9)}$ caused by E. oligosperma have been reported; however, the number of cases remains small. With the advance and wide use of molecular biological search, the number of case reports is expected to increase, which will aid in determin- 
Table 2. Reported cases of deep-seated dermatophytosis caused by E. oligosperma

\begin{tabular}{|c|c|c|c|c|c|c|c|}
\hline $\begin{array}{l}\text { Age/ } \\
\text { Gender }\end{array}$ & Past history & Prior trauma & Duration & Site & Diagnosis & Treatment & Reference \\
\hline $62 / \mathrm{M}$ & $\begin{array}{l}\text { Wegener's granu- } \\
\text { lomatosus }\end{array}$ & No & 8 months & Left elbow & $\begin{array}{l}\text { Olecranon bur- } \\
\text { sitis }\end{array}$ & $\begin{array}{l}\text { Intrabursal injec- } \\
\text { tion of amphoteri- } \\
\text { cin }\end{array}$ & 3 \\
\hline $72 / F$ & $\begin{array}{l}\text { Renal transplanta- } \\
\text { tion }\end{array}$ & No & 3 months & Right leg & $\begin{array}{l}\text { Phaeohyphomy- } \\
\text { cosis }\end{array}$ & Oral itraconazole & 4 \\
\hline $57 / F$ & None & No & 1 year & Left cheek & $\begin{array}{l}\text { Phaeohyphomy- } \\
\text { cosis }\end{array}$ & $\begin{array}{l}\text { Partial surgical } \\
\text { excision topical } \\
\text { terbinafine cream }\end{array}$ & 5 \\
\hline $58 / F$ & $\begin{array}{l}\text { Sjögren syndrome } \\
\text { diabetes mellitus }\end{array}$ & suspected & 5 months & Left hand & $\begin{array}{l}\text { Phaeohyphomy- } \\
\text { cosis }\end{array}$ & Local hyperthermia & This case \\
\hline
\end{tabular}

ing the specific clinical features of various strains and the effective treatment options.

Surgical resection, antifungal therapy, and hyperthermia treatments are current treatment options for phaeohyphomycosis cases. For the present case, we avoided surgical resection because the size of the lesions had poorly defined borders and the patient had underlying medical conditions that could result in surgical complications. Instead, we drained the pus and treated the lesion with hyperthermia, and the size of the lesions rapidly decreased. Since antifungal agents other than amphotericin B are assumed to be ineffective based on MIC values, hyperthermia is still being continued. Based on the experience of the present case, we consider that hyperthermia can be an effective option for treatment of infection caused by E. oligosperma.

\section{References}

1) Zeng JS, De Hoog GS: Exophiala spinifera and its allies: diagnostics from morphology to DNA barcoding. Med Mycol 46: 193-208, 2008.

2) Takahashi $H$, Takahashi-Kyuhachi $H$, Takahashi $Y$, Yarita K, Takayama A, Inomata T, Sano A, Nishimura K, Kamei K: An intrafamilial transmission of Arthroderma benhamiae in Canadian porcupines (Erethizon dorsatum) in a Japanese zoo. Med Mycol 46: 465-473, 2008.
3) Ajello L: The gamut of human infections caused by dematiaceous fungi. Jpn J Med Mycol 22: 1-5, 1981.

4) de Hoog GS, Vicente V, Caligiorne RB, Kantarcioglu S, Tintelnot K, Gerrits van den Ende $A H$, Haase G: Species diversity and polymorphism in the Exophiala spinifera clade containing opportunistic black yeast-like fungi. J Clin Microbiol 41: 4767-4778, 2003.

5) Bossler AD, Richter SS, Chavez AJ, Vogelgesang SA, Sutton DA, Grooters AM, Rinaldi MG, de Hoog GS, Pfaller MA: Exophiala oligosperma causing olecranon bursitis. J Clin Microbiol 41: 4779-4782, 2003.

6) González-López MA, Salesa R, González-Vela MC, Fernández-Llaca H, Val-Bernal JF, Cano J: Subcutaneous phaeohyphomycosis caused by Exophiala oligosperma in a renal transplant recipient. Br J Dermatol 156: 762-764, 2007.

7) Tokuhisa $Y$, Hagiya $Y$, Hiruma M, Nishimura K: Phaeohyphomycosis of the face caused by Exophiala oligosperma. Mycoses 54: e240-e243, 2011.

8) Al-Obaid I, Ahmad S, Khan ZU, Dinesh B, Hejab HM: Catheter-associated fungemia due to Exophiala oligosperma in a leukemic child and review of fungemia cases caused by Exophiala species. Eur $\mathrm{J}$ Clin Microbiol Infect Dis 25: 729-732, 2006.

9) Badali H, Hedayati MT, Bahoosh M, Kasir A, Ghasemi M, Motahari J, Meis JF, De Hoog GS: Exophiala oligosperma involved in a refractory chronic rhinosinusitis. Eur Rev Med Pharmacol Sci 15: 319-323, 2011. 